\title{
Biseparating maps on generalized Lipschitz spaces
}

\author{
by \\ Denny H. Leung (Singapore)
}

\begin{abstract}
Let $X, Y$ be complete metric spaces and $E, F$ be Banach spaces. A bijective linear operator from a space of $E$-valued functions on $X$ to a space of $F$-valued functions on $Y$ is said to be biseparating if $f$ and $g$ are disjoint if and only if $T f$ and $T g$ are disjoint. We introduce the class of generalized Lipschitz spaces, which includes as special cases the classes of Lipschitz, little Lipschitz and uniformly continuous functions. Linear biseparating maps between generalized Lipschitz spaces are characterized as weighted composition operators, i.e., of the form $T f(y)=S_{y}\left(f\left(h^{-1}(y)\right)\right)$ for a family of vector space isomorphisms $S_{y}: E \rightarrow F$ and a homeomorphism $h: X \rightarrow Y$. We also investigate the continuity of $T$ and related questions. Here the functions involved (as well as the metric spaces $X$ and $Y$ ) may be unbounded. Also, the arguments do not require the use of compactification of the spaces $X$ and $Y$.
\end{abstract}

1. Introduction. In his classical treatise, Théorie des Opérations Linéaires [9], Banach proved that the linear isometric structure of the Banach space $C(X)$ of continuous functions on a compact metric space determines the space $X$ up to homeomorphism. The result was generalized by Stone 31] to general compact Hausdorff spaces $X$. Subsequently, Gelfand and Kolmogorov [16] and Kaplansky [25] showed that $X$ is also determined up to homeomorphism by the algebraic structure and the lattice structure of $C(X)$ respectively. In the intervening decades, these types of results have been generalized to many other classes of function spaces and also to spaces of vector-valued functions. The classic monograph [17] considers the relationship between the algebraic structure of spaces of continuous functions on $X$ and the space $X$ itself for general classes of topological spaces. The work [10] uses the Banach-Stone theory of vector-valued continuous functions $C(X, E)$ as a tool to study the Banach space $E$ itself, leading to the theory of $M$ - and $L$-structures of Banach spaces. For the general theory of isometries on Banach spaces, we refer the reader to the two-volume monograph of Fleming and Jamison [11]. For a survey on various aspects of research surrounding Banach-Stone type theorems, see [13].

2010 Mathematics Subject Classification: 46E15, 47B38, 47B33.

Key words and phrases: vector-valued Lipschitz functions, biseparating maps. 
A useful unifying notion that has been introduced into the theory is that of separating or biseparating maps. Two functions $f$ and $g$ defined on the same domain $X$ with values in a vector space are said to be disjoint if for all $x \in X$, either $f(x)=0$ or $g(x)=0$. A map $T$ between vector-valued function spaces is separating (also called disjointness preserving or a Lamperti operator) if $T$ maps disjoint functions to disjoint functions. It is biseparating if $T$ is invertible and both $T$ and $T^{-1}$ are separating. Clearly, algebraic or lattice homomorphisms (isomorphisms) are separating (biseparating). In many instances, isometries between Banach function spaces can also be shown to be biseparating. This explains the interest and amount of work devoted to the characterization of separating or biseparating operators. See, e.g., [1-5, $7,8,15,18,27]$.

The study of Lipschitz spaces can be traced back to de Leeuw [26] and Sherbert [29, 30] for the scalar case, and Johnson [24] for the vector-valued case. A survey on the algebra of Lipschitz functions can be found in [32. Recent work on separating and biseparating maps on Lipschitz spaces and spaces of uniformly continuous functions includes [6, 12, 14, 19, 20, 22, 23. In particular, characterizations of biseparating maps on spaces of bounded Lipschitz or little Lipschitz functions are obtained in [6, 22, 23.

In this paper, we consider spaces of functions determined by the "modulus of continuity" and call such classes generalized Lipschitz spaces. This notion serves to unify the study of spaces of Lipschitz, little Lipschitz and uniformly continuous functions. One of the main aims of this paper is to characterize all biseparating operators between generalized Lipschitz spaces. We make use of a new approach that bypasses the usual compactification procedures, and is rather more closely tied to the metric structure of the underlying spaces. (See $\S 2$.) The second critical ingredient in our argument is the construction of "bump" functions (Lemma 6). Taking advantage of such "bumps" allows us to complete the characterization of biseparating maps as weighted composition operators (Theorem 16). In $\S 3$, we consider questions connected with automatic continuity.

Let $(X, d)$ be a complete metric space and $E$ be a real or complex Banach space. For a function $f: X \rightarrow E$, its modulus of continuity is the function $\omega_{f}:[0, \infty) \rightarrow[0, \infty]$ defined by

$$
\omega_{f}(\varepsilon)=\sup \left\{\left\|f\left(x_{1}\right)-f\left(x_{2}\right)\right\|: d\left(x_{1}, x_{2}\right) \leq \varepsilon\right\} .
$$

Note that $f$ is uniformly continuous on $X$ if and only if $\omega_{f}$ is continuous at 0 . In general, we say that $\sigma:[0, \infty) \rightarrow[0, \infty]$ is a modulus function if $\sigma$ is nondecreasing, $\sigma(0)=0$ and $\sigma$ is continuous at 0 . A nonempty set $\Sigma$ of modulus functions is called a modulus set if:

(MS1) For any $\sigma_{1}, \sigma_{2}$ in $\Sigma$, there exist $\sigma \in \Sigma$ and $K<\infty$ such that $\sigma_{1}+\sigma_{2} \leq K \sigma$. 
(MS2) For every sequence $\left(\sigma_{n}\right)$ in $\Sigma$ and every nonnegative summable real sequence $\left(a_{n}\right)$, there are $\sigma \in \Sigma$ and $K<\infty$ such that $\sum a_{n}\left(\sigma_{n} \wedge 1\right) \leq K \sigma$.

Let $\Sigma$ be a modulus set. Define the generalized Lipschitz space $\operatorname{Lip}_{\Sigma}(X, E)$ to be the set of all functions $f: X \rightarrow E$ such that $\omega_{f} \leq K \sigma$ for some $\sigma \in \Sigma$ and $K<\infty$. Since $\omega_{c f_{1}+f_{2}} \leq|c| \omega_{f_{1}}+\omega_{f_{2}}$, it follows from (MS1) that $\operatorname{Lip}_{\Sigma}(X, E)$ is a vector space. We reiterate that all functions in $\operatorname{Lip}_{\Sigma}(X, E)$ are necessarily uniformly continuous. $\operatorname{Also} \operatorname{Lip}_{\Sigma}(X, E)$ always contains all constant functions. When $E=\mathbb{R}$ or $\mathbb{C}, \operatorname{Lip}_{\Sigma}(X, E)$ is abbreviated to $\operatorname{Lip}_{\Sigma}(X)$. To justify the introduction of this new class of spaces, let us look at a few examples.

EXAMPLES.

(1) If $\Sigma$ consists of the identity function $\sigma(t)=t$ only, then $\operatorname{Lip}_{\Sigma}(X, E)$ is the class $\operatorname{Lip}(X, E)$ of Lipschitz functions. Observe that if $0<\alpha<1$ and we let $X^{\alpha}$ be the space $X$ with the metric $d^{\alpha}$, then $\operatorname{Lip}\left(X^{\alpha}, E\right)$ is the class $\operatorname{Lip}_{\alpha}(X, E)$ of Lipschitz functions (on $(X, d)$ ) of order $\alpha$.

(2) If $\Sigma$ consists of all modulus functions $\sigma$ such that $\sigma(t) \leq t$ for all $t \geq 0$ and $\lim _{t \rightarrow 0} \sigma(t) / t=0$, then $\operatorname{Lip}_{\Sigma}(X, E)$ is the small Lipschitz class $\operatorname{lip}(X, E)$. Again, for $0<\alpha<1, \operatorname{lip}_{\alpha}(X, E)=\operatorname{lip}\left(X^{\alpha}, E\right)$.

(3) If $\Sigma$ is the set of all modulus functions, then $\operatorname{Lip}_{\Sigma}(X, E)$ is the space $\mathcal{U}(X, E)$ of uniformly continuous functions from $X$ to $E$.

(4) If $\Sigma$ is a modulus set and $\Sigma_{b}=\{\sigma \wedge 1: \sigma \in \Sigma\}$, then $\operatorname{Lip}_{\Sigma_{b}}(X, E)$ is the set of all bounded functions in $\operatorname{Lip}_{\Sigma}(X, E)$.

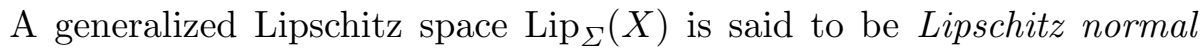
if for every pair of subsets $U, V$ of $X$ with $d(U, V)>0$, there exists $f \in$ $\operatorname{Lip}_{\Sigma}(X), 0 \leq f \leq 1$, such that $f=0$ on $U$ and $f=1$ on $V$. We will say that $\operatorname{Lip}_{\Sigma}(X, E)$ is Lipschitz normal if $\operatorname{Lip}_{\Sigma}(X)$ is. For any metric space $X$, the spaces $\operatorname{Lip}(X), \operatorname{lip}\left(X^{\alpha}\right), 0<\alpha<1$, and $\mathcal{U}(X)$ are Lipschitz normal. Another example is the following: $\operatorname{lip}(\Delta)$ is Lipschitz normal, where $\Delta$ is the Cantor set with the usual metric. In this paper, all generalized Lipschitz spaces considered are assumed to be Lipschitz normal.

If $f$ belongs to $\operatorname{Lip}_{\Sigma}(X, E)$, let $C(f)$ be the set $\{x \in X: f(x) \neq 0\}$ and denote its closure by $\bar{C}(f)$. If $\left(Y, d^{\prime}\right)$ is a complete metric space, $F$ is a Banach space and $\Sigma^{\prime}$ is a modulus set, we may define the $\operatorname{space}_{\operatorname{Lip}_{\Sigma^{\prime}}}(Y, F)$ as above. A linear map $T: \operatorname{Lip}_{\Sigma}(X, E) \rightarrow \operatorname{Lip}_{\Sigma^{\prime}}(Y, F)$ is said to be biseparating if $T$ is a bijection and, for all $f, g \in \operatorname{Lip}_{\Sigma}(X, E)$,

$$
C(f) \cap C(g)=\emptyset \quad \text { if and only if } \quad C(T f) \cap C(T g)=\emptyset .
$$

2. The Boolean algebra of closures of open sets. Let $X$ be a complete metric space with metric $d$. Denote by $\mathcal{D}(X)$ the collection of all 
subsets $A$ of $X$ such that $A=\overline{\operatorname{int} A}$. Equivalently, $A \in \mathcal{D}(X)$ if and only if $A$ is the closure of an open subset of $X$. In particular, $\bar{C}(f) \in \mathcal{D}(X)$ for every $f \in \operatorname{Lip}_{\Sigma}(X, E)$. Moreover, $\mathcal{D}(X)$ is a Boolean algebra under the order of set inclusion, with lattice operations

$$
A \vee B=A \cup B \quad \text { and } \quad A \wedge B=\overline{\operatorname{int} A \cap \operatorname{int} B}
$$

for all $A, B \in \mathcal{D}(X)$. The 0 and 1 elements of $\mathcal{D}(X)$ are $\emptyset$ and $X$ respectively; the complement of $A \in \mathcal{D}(X)$ is

$$
\neg A=\overline{A^{c}},
$$

where $A^{c}$ is the set-theoretic complement of $A$. For basic properties of Boolean algebras we refer the reader to [28. We begin with a simple but fundamental observation.

Proposition 1. Let $\varphi$ be a function in $\operatorname{Lip}_{\Sigma}(X)$ with values in $[0,1]$ and let $f \in \operatorname{Lip}_{\Sigma}(X, E)$ be such that $\|f(x)\| \leq M$ for all $x \in C(\varphi)$. Then $\varphi f \in \operatorname{Lip}_{\Sigma}(X, E)$ and $\omega_{\varphi f} \leq \omega_{f}+M \omega_{\varphi}$.

Proof. Suppose that $d\left(x_{1}, x_{2}\right) \leq \varepsilon$. If neither $x_{1}$ nor $x_{2}$ lies in $C(\varphi)$, then $\left\|(\varphi f)\left(x_{1}\right)-(\varphi f)\left(x_{2}\right)\right\|=0$. Otherwise, we may assume that $x_{2} \in C(\varphi)$ and hence $\left\|f\left(x_{2}\right)\right\| \leq M$. Therefore,

$$
\begin{aligned}
\left\|(\varphi f)\left(x_{1}\right)-(\varphi f)\left(x_{2}\right)\right\| & \leq\left|\varphi\left(x_{1}\right)\right|\left\|f\left(x_{1}\right)-f\left(x_{2}\right)\right\|+\left|\varphi\left(x_{1}\right)-\varphi\left(x_{2}\right)\right|\left\|f\left(x_{2}\right)\right\| \\
& \leq \omega_{f}(\varepsilon)+M \omega_{\varphi}(\varepsilon) .
\end{aligned}
$$

The next lemma is similar to Lemma 4.2 in [1].

Lemma 2. Let $T: \operatorname{Lip}_{\Sigma}(X, E) \rightarrow \operatorname{Lip}_{\Sigma^{\prime}}(Y, F)$ be a biseparating map. If $f, g \in \operatorname{Lip}_{\Sigma}(X, E)$ and $\bar{C}(f) \subseteq \bar{C}(g)$, then $C(T f) \subseteq \bar{C}(T g)$.

Proof. Suppose that $y \notin \bar{C}(T g)$. There exists $\varepsilon>0$ so that $B(y, \varepsilon) \cap$ $\bar{C}(T g)=\emptyset$ and that $T f$ is bounded on $B(y, \varepsilon)$. Let $\psi$ be a $[0,1]$-valued function in $\operatorname{Lip}_{\Sigma^{\prime}}(Y)$ so that $\psi=1$ on $B(y, \varepsilon / 2)$ and $\psi=0$ outside $B(y, \varepsilon)$. Then $\psi T f \in \operatorname{Lip}_{\Sigma^{\prime}}(Y, F)$ by Proposition 1 and $C(\psi T f) \cap C(T g)=\emptyset$. Thus $C\left(T^{-1}(\psi T f)\right) \cap C(g)=\emptyset$. Since $C\left(T^{-1}(\psi T f)\right)$ is an open set, it follows that $C\left(T^{-1}(\psi T f)\right) \cap \bar{C}(g)=\emptyset$, and hence $C\left(T^{-1}(\psi T f)\right) \cap C(f)=\emptyset$. Therefore, $C(\psi T f) \cap C(T f)=\emptyset$. In particular, since $\psi(y) \neq 0$, we must have $T f(y)=0$. So $y \notin C(T f)$.

Lemma 3. For each open subset $U$ of $X$, there exists $f \in \operatorname{Lip}_{\Sigma}(X, E)$ such that $C(f)=U$.

Proof. For each $n \in \mathbb{N}$, let $U_{n}$ be the set of all $x \in X$ such that $d\left(x, U^{c}\right) \geq$ $1 / n$. Since $\operatorname{Lip}_{\Sigma}(X, E)$ is Lipschitz normal, there exists $\varphi_{n} \in \operatorname{Lip}_{\Sigma}(X)$ with values in $[0,1]$ so that $\varphi_{n}=0$ on $U^{c}$ and $\varphi_{n}=1$ on $U_{n}$. Take $\sigma_{n} \in \Sigma$ and $K_{n}<\infty$ so that $\omega_{\varphi_{n}} \leq K_{n} \sigma_{n}$. Note that $\omega_{\varphi_{n}} \leq 1$ as well. So, by redefining the constant $K_{n}$ if necessary, we may assume that $\omega_{\varphi_{n}} \leq K_{n}\left(\sigma_{n} \wedge 1\right)$. The 
function $\varphi=\sum \varphi_{n} /\left(n^{2}\left(K_{n}+1\right)\right)$ converges on $X$. Also,

$$
\omega_{\varphi} \leq \sum \frac{\omega_{\varphi_{n}}}{n^{2}\left(K_{n}+1\right)} \leq \sum \frac{\sigma_{n} \wedge 1}{n^{2}} \leq K \sigma
$$

for some $\sigma \in \Sigma$ and $K<\infty$ by condition (MS2) in the definition of modulus sets. Thus $\varphi \in \operatorname{Lip}_{\Sigma}(X)$. Clearly, $C(\varphi)=U$. Finally, choose any nonzero $u \in E$ and $f(x)=\varphi(x) u$ is a function with the desired properties.

Proposition 4. Let $T: \operatorname{Lip}_{\Sigma}(X, E) \rightarrow \operatorname{Lip}_{\Sigma^{\prime}}(Y, F)$ be a biseparating map. For each $A \in \mathcal{D}(X)$, let $\theta(A)=\bar{C}(T f)$ for some $f \in \operatorname{Lip}_{\Sigma}(X, E)$ such that $C(f)=\operatorname{int} A$. Then $\theta$ is a well-defined Boolean isomorphism from $\mathcal{D}(X)$ onto $\mathcal{D}(Y)$. Moreover, for any $f \in \operatorname{Lip}_{\Sigma}(X, E)$ and any $A \in \mathcal{D}(X)$, $f=0$ on $A$ if and only if $T f=0$ on $\theta(A)$.

Proof. The fact that $\theta$ is well defined follows from Lemmas 2 and 3 . By Lemma 2, $\theta$ preserves order. Analogously, we can define $\tau: \mathcal{D}(Y) \rightarrow \mathcal{D}(X)$ by $\tau(B)=\bar{C}\left(T^{-1} g\right)$ for some $g \in \operatorname{Lip}_{\Sigma^{\prime}}(Y, F)$ such that $C(g)=\operatorname{int} B$. If $A \in \mathcal{D}(X)$ and $f \in \operatorname{Lip}_{\Sigma}(X, E)$ with $C(f)=\operatorname{int} A$, then $\theta(A)=\bar{C}(T f)$. Let $g \in \operatorname{Lip}_{\Sigma^{\prime}}(Y, F)$ be such that $C(g)=\operatorname{int} \theta(A)$. By Lemma 2 applied to $T^{-1}$, $\bar{C}(f)=\bar{C}\left(T^{-1} g\right)$. Thus $A=\bar{C}(f)=\tau(\theta(A))$. Similarly, $\theta(\tau(B))=B$ for all $B \in \mathcal{D}(Y)$. Hence $\tau=\theta^{-1}$. Since both $\theta$ and $\theta^{-1}$ are order preserving, $\theta$ is a Boolean isomorphism.

If $f \in \operatorname{Lip}_{\Sigma}(X, E)$ and $f=0$ on $A \in \mathcal{D}(X)$, then $C(f) \cap C\left(f^{\prime}\right)=\emptyset$ for any $f^{\prime} \in \operatorname{Lip}_{\Sigma}(X, E)$ with $C\left(f^{\prime}\right)=\operatorname{int} A$. Hence $C(T f) \cap C\left(T f^{\prime}\right)=\emptyset$. By continuity of $T f, T f=0$ on $\bar{C}\left(T f^{\prime}\right)=\theta(A)$. The converse follows by symmetry.

3. Characterization of biseparating maps. Let $X$ and $Y$ be complete metric spaces, $E$ and $F$ be Banach spaces, and $\Sigma$ and $\Sigma^{\prime}$ be two modulus sets. The closed unit ball of $F$ is denoted by $B_{F}$. We begin with an easy observation.

Lemma 5. For any $a>0$, the retraction $r: F \rightarrow a B_{F}$ defined by

$$
r(v)= \begin{cases}v & \text { if } v \in a B_{F}, \\ a v /\|v\| & \text { otherwise, }\end{cases}
$$

is a Lipschitz map with $\omega_{r}(t) \leq 2 t$.

Lemma 6. Let $g$ be a function in $\operatorname{Lip}_{\Sigma^{\prime}}(Y, F)$. For all $a>0$ and all $b \geq 2 a$, there is a function $\tilde{g} \in \operatorname{Lip}_{\Sigma^{\prime}}(Y, F)$ with $\omega_{\tilde{g}} \leq 3 \omega_{g}$ such that

$$
\tilde{g}(y)= \begin{cases}g(y) & \text { if }\|g(y)\| \leq a \\ 0 & \text { if }\|g(y)\| \geq b .\end{cases}
$$

Proof. Let $r: F \rightarrow a B_{F}$ be the retraction defined in Lemma 5. Then $\omega_{r \circ g} \leq 2 \omega_{g}$ and $r \circ g$ is bounded in norm by $a$. For any $b \geq 2 a$, the function 
$\gamma:[0, \infty) \rightarrow[0,1]$ defined by

$$
\gamma(t)= \begin{cases}1 & \text { if } t \in[0, a], \\ \frac{b-t}{b-a} & \text { if } t \in(a, b), \\ 0 & \text { if } t \in[b, \infty)\end{cases}
$$

satisfies $\omega_{\gamma}(t) \leq t / a$. Let $\tilde{g}(y)=\gamma(\|g(y)\|) r(g(y))$. Clearly, $\tilde{g}(y)=g(y)$ if $\|g(y)\| \leq a$ and 0 if $\|g(y)\| \geq b$. For all $y_{1}, y_{2} \in Y$ with $d^{\prime}\left(y_{1}, y_{2}\right) \leq \varepsilon$,

$$
\begin{aligned}
\left\|\tilde{g}\left(y_{1}\right)-\tilde{g}\left(y_{2}\right)\right\| \leq & \gamma\left(\left\|g\left(y_{1}\right)\right\|\right)\left\|r\left(g\left(y_{1}\right)\right)-r\left(g\left(y_{2}\right)\right)\right\| \\
& +\left|\gamma\left(\left\|g\left(y_{1}\right)\right\|\right)-\gamma\left(\left\|g\left(y_{2}\right)\right\|\right)\right|\left\|r\left(g\left(y_{2}\right)\right)\right\| \\
\leq & \omega_{r \circ g}(\varepsilon)+\frac{1}{a}\left|\left\|g\left(y_{1}\right)\right\|-\left\|g\left(y_{2}\right)\right\|\right| a \leq 3 \omega_{g}(\varepsilon) .
\end{aligned}
$$

Thus $\omega_{\tilde{g}} \leq 3 \omega_{g}$ and $\tilde{g} \in \operatorname{Lip}_{\Sigma^{\prime}}(Y, F)$.

LEMma 7. Let $\left(f_{n}\right)$ be a pairwise disjoint sequence of functions from $X$ into E. Assume that there is a modulus function $\sigma$ such that $\omega_{f_{n}} \leq \sigma$ for all $n$. Then the pointwise sum $f=\sum f_{n}$ satisfies $\omega_{f} \leq 2 \sigma$.

Proof. For any $x_{1}, x_{2} \in X$, either there exists $n_{1}$ such that $f\left(x_{i}\right)=$ $f_{n_{1}}\left(x_{i}\right), i=1,2$, or there are $n_{1}$ and $n_{2}$ so that $f\left(x_{i}\right)=\left(f_{n_{1}}+f_{n_{2}}\right)\left(x_{i}\right)$, $i=1,2$. It follows that $\omega_{f} \leq 2 \sup _{n} \omega_{f_{n}} \leq 2 \sigma$.

For the rest of the section, we consider a linear biseparating map $T$ : $\operatorname{Lip}_{\Sigma}(X, E) \rightarrow \operatorname{Lip}_{\Sigma^{\prime}}(Y, F)$. Let $\theta$ be the associated Boolean isomorphism from Proposition 4 . If $u$ is a vector in $E$ or $F$, denote by $\hat{u}$ the constant function (defined on $X$ or $Y$ ) with value $u$. The next proposition is a key to subsequent arguments.

Proposition 8. For any $x_{0} \in X$, there exists $f \in \operatorname{Lip}_{\Sigma}(X, E)$ such that $f\left(x_{0}\right) \neq 0$ and $T f$ is bounded on $Y$.

Proof. Suppose that the proposition fails. We have $x_{0} \in X$ so that $T f$ is unbounded whenever $f\left(x_{0}\right) \neq 0$. Pick any $u \in E \backslash\{0\}$ and let $g=T \hat{u}$. First we need two lemmas.

Lemma 9. For all $a>0$ and all $\varepsilon>0, \theta^{-1}(\overline{\{\|g\|>a\}}) \wedge \overline{B\left(x_{0}, \varepsilon\right)} \neq 0$.

Proof. Suppose that $\theta^{-1}(\overline{\{\|g\|>a\}}) \wedge \overline{B\left(x_{0}, \varepsilon\right)}=0$ for some $a, \varepsilon>0$. Let $V=\neg(\overline{\{\|g\|>a\}})$. Then

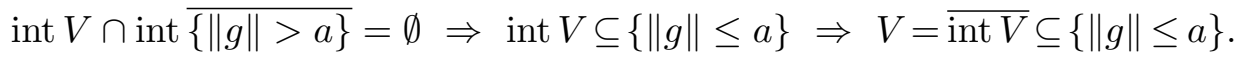

Since $g$ is uniformly continuous, we have $d^{\prime}(V,\{\|g\| \geq 2 a\})>0$. Hence there exists $\psi \in \operatorname{Lip}_{\Sigma^{\prime}}(Y)$ such that $0 \leq \psi \leq 1, \psi=1$ on $V$ and $\psi=0$ on $\{\|g\| \geq 2 a\}$. Note that $g$ is bounded on $C(\psi)$ and hence $\psi g \in \operatorname{Lip}_{\Sigma^{\prime}}(Y, F)$ by Proposition 1. As $\psi g=g$ on $V$, we have $T^{-1}(\psi g)=T^{-1} g=\hat{u}$ on $\theta^{-1}(V)$ by Proposition 4 . From $\theta^{-1}(\overline{\{\|g\|>a\}}) \wedge \overline{B\left(x_{0}, \varepsilon\right)}=0$, and keeping 
in mind the definition of $V$, we see that $\overline{B\left(x_{0}, \varepsilon\right)} \subseteq \theta^{-1}(V)$. In particular, $T^{-1}(\psi g)\left(x_{0}\right)=u \neq 0$. By assumption, $\psi g$ is unbounded on $Y$. But this is clearly false since $\|(\psi g)(y)\| \leq 2 a$ for all $y \in Y$.

Lemma 10. For all $a>0$ and all $\varepsilon>0$, there exists $b>a$ so that $\theta^{-1}(\overline{\{\|g\| \in(a, b)\}}) \wedge \overline{B\left(x_{0}, \varepsilon\right)} \neq 0$.

Proof. For each $n \in \mathbb{N}$, let $V_{n}=\overline{\{\|g\| \in(a, a+n)\}}$. The sequence $\left(V_{n}\right)$ increases to $\overline{\{\|g\|>a\}}$ in $\mathcal{D}(Y)$. Hence $\left(\theta^{-1}\left(V_{n}\right)\right)$ increases to $\theta^{-1}(\overline{\{\|g\|>a\}})$ in $\mathcal{D}(X)$. If the lemma fails, $\theta^{-1}\left(V_{n}\right) \subseteq \neg\left(\overline{B\left(x_{0}, \varepsilon\right)}\right)$ for all $n$ and thus $\theta^{-1}(\overline{\{\|g\|>a\}}) \subseteq \neg\left(\overline{B\left(x_{0}, \varepsilon\right)}\right)$, contrary to Lemma 9 .

We now continue the proof of Proposition 8 . Let $\left(\varepsilon_{n}\right)$ be a positive null sequence and set $a_{1}=1$. By Lemma 6 , there exist $b_{1}>a_{1}$ and $\tilde{g}_{1} \in \operatorname{Lip}_{\Sigma^{\prime}}(Y, F)$ with $\omega_{\tilde{g}_{1}} \leq 3 \omega_{g}$ such that

$$
\tilde{g}_{1}(y)= \begin{cases}g(y) & \text { if }\|g(y)\| \leq a_{1}, \\ 0 & \text { if }\|g(y)\| \geq b_{1} .\end{cases}
$$

In general, after $a_{n}, b_{n}$ have been determined, use Lemma 10 to choose $a_{n+1}>b_{n}$ such that $\theta^{-1}\left(\overline{\left\{\|g\| \in\left(b_{n}, a_{n+1}\right)\right\}}\right) \wedge \overline{B\left(x_{0}, \varepsilon_{n}\right)} \neq 0$. Then apply Lemma 6 to obtain $b_{n+1}>a_{n+1}$ and $\tilde{g}_{n+1} \in \operatorname{Lip}_{\Sigma^{\prime}}(Y, F)$ with $\omega_{\tilde{g}_{n+1}} \leq 3 \omega_{g}$ so that

$$
\tilde{g}_{n+1}(y)= \begin{cases}g(y) & \text { if }\|g(y)\| \leq a_{n+1}, \\ 0 & \text { if }\|g(y)\| \geq b_{n+1}\end{cases}
$$

For each $n$, let $G_{n}=\tilde{g}_{2 n}-\tilde{g}_{2 n-1}$. Then $G_{n} \in \operatorname{Lip}_{\Sigma^{\prime}}(Y, F)$ and $\omega_{G_{n}} \leq 6 \omega_{g}$. Also, $G_{n}(y)=0$ if $\|g(y)\| \notin\left(a_{2 n-1}, b_{2 n}\right)$ and $G_{n}(y)=g(y)$ if $\|g(y)\| \in$ $\left[b_{2 n-1}, a_{2 n}\right]$. In particular, $C\left(G_{n}\right)$ and $C\left(G_{m}\right)$ are disjoint if $n \neq m$. Thus the pointwise sum $G=\sum G_{2 m-1}$ is well defined and $\omega_{G} \leq 12 \omega_{g}$ by Lemma 7 Hence $G \in \operatorname{Lip}_{\Sigma^{\prime}}(Y, F)$. For each $m$, let $V_{m}=\overline{\left\{\|g\| \in\left(b_{2 m-1}, a_{2 m}\right)\right\}}$. By the choice of $a_{2 m}$, one can find $x_{m} \in \theta^{-1}\left(V_{m}\right) \cap \overline{B\left(x_{0}, \varepsilon_{2 m-1}\right)}$. Now $G=$ $G_{2 m-1}=g$ on $V_{2 m-1}$ and $G=0$ on $V_{2 m}$ for all $m$. Hence $T^{-1} G=T^{-1} g=\hat{u}$ on $\theta^{-1}\left(V_{2 m-1}\right)$ and $T^{-1} G=0$ on $\theta^{-1}\left(V_{2 m}\right)$ by Proposition 4. In particular, $T^{-1} G\left(x_{2 m-1}\right)=u \neq 0$ and $T^{-1} G\left(x_{2 m}\right)=0$ for all $m$. Since the sequence $\left(x_{m}\right)$ converges to $x_{0}$ and $T^{-1} G$ is continuous, we have reached a contradiction. This completes the proof of Proposition 8 .

Lemma 11. For any $x_{0} \in X, \bigcap_{\varepsilon>0} \theta\left(\overline{B\left(x_{0}, \varepsilon\right)}\right)$ contains at most one point.

Proof. Suppose on the contrary that $y_{1}$ and $y_{2}$ are distinct points in the intersection. Let $f \in \operatorname{Lip}_{\Sigma}(X, E)$ be such that $f\left(x_{0}\right) \neq 0$ and set $g=T f$. Choose $\delta>0$ so that $d^{\prime}\left(y_{1}, y_{2}\right)>3 \delta$ and that $g$ is bounded on $B\left(y_{1}, 2 \delta\right)$. Pick $\psi \in \operatorname{Lip}_{\Sigma^{\prime}}(Y)$ so that $\psi=1$ on $\overline{B\left(y_{1}, \delta\right)}$ and $\psi=0$ outside $B\left(y_{1}, 2 \delta\right)$. Since $g$ is bounded on $C(\psi), \psi g \in \operatorname{Lip}_{\Sigma^{\prime}}(Y, F)$. As $\psi g=g$ on $\overline{B\left(y_{1}, \delta\right)}, T^{-1}(\psi g)=f$ on $\theta^{-1}\left(\overline{B\left(y_{1}, \delta\right)}\right)$. Similarly, $T^{-1}(\psi g)=0$ on $\theta^{-1}\left(\overline{B\left(y_{2}, \delta\right)}\right)$. For $i=1,2$ and 
any $\varepsilon>0, \theta\left(\overline{B\left(x_{0}, \varepsilon\right)}\right) \wedge \overline{B\left(y_{i}, \delta\right)} \neq 0$ and hence $\overline{B\left(x_{0}, \varepsilon\right)} \wedge \theta^{-1}\left(\overline{B\left(y_{i}, \delta\right)}\right) \neq 0$. By continuity of $T^{-1}(\psi g)$ and $f$, we conclude that $T^{-1}(\psi g)\left(x_{0}\right)=f\left(x_{0}\right)$ and $T^{-1}(\psi g)\left(x_{0}\right)=0$, thus reaching a contradiction.

Lemma 12. Assume that $x_{0} \in X$ is an accumulation point. Let $\left(U_{n}\right)=$ $\left(\overline{B\left(x_{n}, \varepsilon_{n}\right)}\right)$ be a sequence of pairwise disjoint sets, where $\left(x_{n}\right)$ converges to $x_{0}$ and $\left(\varepsilon_{n}\right)$ is a positive null sequence. If $\left(y_{n}\right)$ is a sequence such that $y_{n} \in \operatorname{int} \theta\left(U_{n}\right)$ for each $n$, then $\left(y_{n}\right)$ has a Cauchy subsequence.

Proof. If the lemma fails, by passing to a subsequence if necessary, we may assume that there exists $\delta>0$ such that $d^{\prime}\left(y_{m}, y_{n}\right)>3 \delta$ whenever $m \neq n$. There exists a $[0,1]$-valued $\psi \in \operatorname{Lip}_{\Sigma^{\prime}}(Y)$ such that $\psi=1$ on $\overline{B\left(y_{n}, \delta\right)}$ for all odd $n$ and $\psi=0$ on $\overline{B\left(y_{n}, \delta\right)}$ for all even $n$. According to Proposition 8, there exists $f \in \operatorname{Lip}_{\Sigma}(X, E)$ such that $f\left(x_{0}\right) \neq 0$ and $T f$ is bounded on $Y$. Then $g=\psi T f \in \operatorname{Lip}_{\Sigma^{\prime}}(Y, F)$. Now $g=T f$ on $\overline{B\left(y_{n}, \delta\right)}$ for all odd $n$ and $g=0$ on $\overline{B\left(y_{n}, \delta\right)}$ for all even $n$. Hence $T^{-1} g=f$ on $\theta^{-1}\left(\overline{B\left(y_{n}, \delta\right)}\right)$ for all odd $n$ and $T^{-1} g=0$ on $\theta^{-1}\left(\overline{B\left(y_{n}, \delta\right)}\right)$ for all even $n$. Since $\theta\left(U_{n}\right) \wedge$ $\overline{B\left(y_{n}, \delta\right)} \neq 0$ for all $n, U_{n} \wedge \theta^{-1}\left(\overline{B\left(y_{n}, \delta\right)}\right) \neq 0$ for all $n$. Therefore, we can find a sequence $\left(z_{n}\right)$ such that $z_{n} \in U_{n}$ for all $n, T^{-1} g\left(z_{n}\right)=f\left(z_{n}\right)$ for odd $n$ and $T^{-1} g\left(z_{n}\right)=0$ for even $n$. This is impossible since $T^{-1} g$ and $f$ are continuous, $f\left(x_{0}\right) \neq 0$ and $\left(z_{n}\right)$ converges to $x_{0}$.

Proposition 13. For any $x_{0} \in X, \bigcap_{\varepsilon>0} \theta\left(\overline{B\left(x_{0}, \varepsilon\right)}\right)$ contains exactly one point.

Proof. In view of Lemma 11, it suffices to prove that the intersection in question is nonempty. If $x_{0}$ is an isolated point, then $\left\{x_{0}\right\} \in \mathcal{D}(X)$ and $\theta\left(\left\{x_{0}\right\}\right) \subseteq \bigcap_{\varepsilon>0} \theta\left(\overline{B\left(x_{0}, \varepsilon\right)}\right)$. So the proposition holds in this case.

Assume that $x_{0}$ is an accumulation point. Let $\left(U_{n}\right)=\left(\overline{B\left(x_{n}, \varepsilon_{n}\right)}\right)$ be a sequence of pairwise disjoint sets, where $\left(x_{n}\right)$ converges to $x_{0}$ and $\left(\varepsilon_{n}\right)$ is a positive null sequence. Pick a sequence $\left(y_{n}\right)$ with $y_{n} \in \operatorname{int} \theta\left(U_{n}\right)$ for each $n$. By Lemma 12, $\left(y_{n}\right)$ has a Cauchy subsequence. Relabeling, we may assume that $\left(y_{n}\right)$ is Cauchy and hence converges to some $y_{0} \in Y$. For any $\varepsilon>0$, there exists $n_{0}$ such that $U_{n} \subseteq \overline{B\left(x_{0}, \varepsilon\right)}$ for all $n \geq n_{0}$. Thus $y_{n} \in \theta\left(U_{n}\right) \subseteq$ $\theta\left(\overline{B\left(x_{0}, \varepsilon\right)}\right)$ for all $n \geq n_{0}$. Since the latter set is closed, $y_{0} \in \theta\left(\overline{B\left(x_{0}, \varepsilon\right)}\right)$.

Define $h\left(x_{0}\right)$ to be the unique point in $\bigcap_{\varepsilon>0} \theta\left(\overline{B\left(x_{0}, \varepsilon\right)}\right)$ for all $x_{0} \in X$. Similarly, we may define $k: Y \rightarrow X$ by setting $k\left(y_{0}\right)$ to be the unique point in $\bigcap_{\delta>0} \theta^{-1}\left(\overline{B\left(y_{0}, \delta\right)}\right)$.

Proposition 14. The map $h$ is a homeomorphism whose inverse is $k$.

Proof. Suppose that $x_{0} \in X$ and $h\left(x_{0}\right)=y_{0}$. For any $\varepsilon, \delta>0, \overline{B\left(y_{0}, \delta\right)} \wedge$ $\theta\left(\overline{B\left(x_{0}, \varepsilon\right)}\right) \neq 0$ and hence $\theta^{-1}\left(\overline{B\left(y_{0}, \delta\right)}\right) \wedge \overline{B\left(x_{0}, \varepsilon\right)} \neq 0$. In particular, for any $\delta>0$, we can find $x_{n} \in \theta^{-1}\left(\overline{B\left(y_{0}, \delta\right)}\right) \wedge \overline{B\left(x_{0}, 1 / n\right)}$ for each $n$. Since 
$\theta^{-1}\left(\overline{B\left(y_{0}, \delta\right)}\right)$ is closed, $x_{0} \in \theta^{-1}\left(\overline{B\left(y_{0}, \delta\right)}\right)$. As $\delta>0$ is arbitrary, this shows that $k\left(y_{0}\right)=x_{0}$. By symmetry, $h\left(k\left(y_{0}\right)\right)=y_{0}$ for all $y_{0} \in Y$.

It remains to prove the continuity of $h$. The continuity of $k$ follows by symmetry. Let $x_{0}$ be a point in $X$. Since $h$ is trivially continuous at an isolated point, we may assume that $x_{0}$ is an accumulation point. Let $\left(x_{n}\right)$ be a pairwise distinct sequence converging to $x_{0}$. Choose a positive null sequence $\left(\varepsilon_{n}\right)$ so that $\left(U_{n}\right)=\left(\overline{B\left(x_{n}, \varepsilon_{n}\right)}\right)$ is pairwise disjoint. For each $n$, $h\left(x_{n}\right) \in \theta\left(U_{n}\right)=\overline{\operatorname{int} \theta\left(U_{n}\right)}$. Hence there exists $y_{n} \in \operatorname{int} \theta\left(U_{n}\right)$ so that $d^{\prime}\left(y_{n}, h\left(x_{n}\right)\right)<1 / n$. By Lemma 12, $\left(y_{n}\right)$ has a subsequence converging to a point $y_{0}$ in $\bigcap_{\varepsilon>0} \theta\left(\overline{B\left(x_{0}, \varepsilon\right)}\right)$. Consequently, $\left(h\left(x_{n}\right)\right)$ has a subsequence that converges to $y_{0}$. By Lemma 11, $y_{0}=h\left(x_{0}\right)$. The continuity of $h$ at $x_{0}$ follows.

Observe that if $f=0$ on an open set $U$ containing $x_{0}$, then $f=0$ on $\overline{B\left(x_{0}, \varepsilon\right)}$ for some $\varepsilon>0$ and hence $T f=0$ on $\theta\left(\overline{B\left(x_{0}, \varepsilon\right)}\right)$. In particular, $T f\left(h\left(x_{0}\right)\right)=0$.

Proposition 15. If $f \in \operatorname{Lip}_{\Sigma}(X, E)$ and $f\left(x_{0}\right)=0$, then $T f\left(h\left(x_{0}\right)\right)=0$.

Proof. By the observation preceding the proposition, we only need to consider the case where $x_{0}$ is an accumulation point of $C(f)$. Suppose $z$ belongs to $C(f)$. By Lemma 6, there are functions $g_{1}, g_{2}: X \rightarrow E$ with $\omega_{g_{i}} \leq 3 \omega_{f}, i=1,2$, so that

$$
g_{1}(x)= \begin{cases}f(x) & \text { if }\|f(x)\| \leq 2\|f(z)\|, \\ 0 & \text { if }\|f(x)\| \geq 4\|f(z)\|,\end{cases}
$$

and

$$
g_{2}(x)= \begin{cases}f(x) & \text { if }\|f(x)\| \leq\|f(z)\| / 4, \\ 0 & \text { if }\|f(x)\| \geq\|f(z)\| / 2 .\end{cases}
$$

Set $g=g_{1}-g_{2}$. Then $\omega_{g} \leq 6 \omega_{f}$ and

$$
g(x)= \begin{cases}f(x) & \text { if }\|f(x)\| \in[\|f(z)\| / 2,2\|f(z)\|], \\ 0 & \text { if }\|f(x)\| \notin[\|f(z)\| / 4,4\|f(z)\|] .\end{cases}
$$

Let $\left(x_{n}\right)$ be a sequence in $C(f)$ converging to $x_{0}$ so that $\left\|f\left(x_{n+1}\right)\right\| \leq$ $16\left\|f\left(x_{n}\right)\right\|$ for all $n$. For each $n$, let $f_{n}$ be the function $g$ described above with $z=x_{2 n-1}$. By Lemma 7, $\tilde{f}=\sum f_{n}$ belongs to $\operatorname{Lip}_{\Sigma}(X, E)$. For each $n$,

$$
A_{n}=\left\{x \in X:\left\|f\left(x_{n}\right)\right\| / 2<\|f(x)\|<2\left\|f\left(x_{n}\right)\right\|\right\}
$$

is an open neighborhood of $x_{n}$. Furthermore, $\tilde{f}=f$ on $\overline{A_{n}}$ if $n$ is odd and $\tilde{f}=0$ on $\overline{A_{n}}$ if $n$ is even. By Proposition $4, T \tilde{f}=T f$ on $\theta\left(\overline{A_{n}}\right)$ for odd $n$ and $T \tilde{f}=0$ on $\theta\left(\overline{A_{n}}\right)$ for even $n$. In particular, $T \tilde{f}\left(h\left(x_{n}\right)\right)=T f\left(h\left(x_{n}\right)\right)$ if $n$ 
is odd and 0 if $n$ is even. By continuity of $T \tilde{f}, T f$ and $h$, we have

$$
\begin{aligned}
T f\left(h\left(x_{0}\right)\right) & =\lim T f\left(h\left(x_{2 n-1}\right)\right)=\lim T \tilde{f}\left(h\left(x_{2 n-1}\right)\right) \\
& =T \tilde{f}\left(h\left(x_{0}\right)\right)=\lim T \tilde{f}\left(h\left(x_{2 n}\right)\right)=0 .
\end{aligned}
$$

The following is the main result of this section. It includes as a special case the result of [6] characterizing biseparating maps between spaces of bounded Lipschitz functions.

Theorem 16. Let $X, Y$ be complete metric spaces and $E, F$ be Banach spaces. Suppose that $\operatorname{Lip}_{\Sigma}(X, E)$ and $\operatorname{Lip}_{\Sigma^{\prime}}(Y, F)$ are generalized Lipschitz spaces that are Lipschitz normal. If $T: \operatorname{Lip}_{\Sigma}(X, E) \rightarrow \operatorname{Lip}_{\Sigma^{\prime}}(Y, F)$ is a linear biseparating map, then there exist a homeomorphism $h: X \rightarrow Y$ and, for each $y \in Y$, a vector space isomorphism $S_{y}: E \rightarrow F$ such that

$$
T f(y)=S_{y}\left(f\left(h^{-1}(y)\right)\right) \quad \text { for all } y \in Y \text {. }
$$

Proof. Let $h: X \rightarrow Y$ be defined as above. Then $h$ is a homeomorphism by Proposition 14. Define $S_{y}: E \rightarrow F$ by $S_{y} u=T \hat{u}(y)$ for all $y \in Y$. If $f \in \operatorname{Lip}_{\Sigma}(X, E)$ and $y \in Y$, then $(f-\hat{u})\left(h^{-1}(y)\right)=0$, where $u=f\left(h^{-1}(y)\right)$. Therefore, $T f(y)=T \hat{u}(y)$ by Proposition 15. Thus (1) holds. The linearity of $S_{y}$ follows from that of $T$. If $v \in F$, there exists $f$ such that $T f=\hat{v}$. Hence, for any $y$, taking $u=f\left(h^{-1}(y)\right)$, we find that $S_{y} u=v$. This shows that each $S_{y}$ is onto. Finally, if $S_{y} u=0$, then $T \hat{u}(y)=0$. Applying Proposition 15 to $T^{-1}$ and $h^{-1}$, we find that $u=T^{-1} T \hat{u}\left(h^{-1}(y)\right)=0$. Thus $S_{y}$ is one-to-one.

4. Continuity. In this section, let $T: \operatorname{Lip}_{\Sigma}(X, E) \rightarrow \operatorname{Lip}_{\Sigma^{\prime}}(Y, F)$ be a biseparating map. Thus $T$ has the form given in (1) of Theorem 16, where $h$ is a homeomorphism and $S_{y}$ is a vector space isomorphism for all $y \in Y$. We investigate the continuity properties of the family $\left(S_{y}\right)$ and of the operator $T$ with respect to suitable topologies. We also consider the metric properties of the mapping $h$.

Proposition 17. If $y_{0}$ is an accumulation point in $Y$, then $S_{y_{0}}$ is a bounded linear operator. Furthermore, if

$$
\sup _{\sigma \in \Sigma^{\prime}} \sigma(\varepsilon)<\infty \quad \text { for all } \varepsilon \geq 0,
$$

then $S_{y}$ is bounded at all $y \in Y$ except for finitely many isolated points of $Y$.

Proof. Assume on the contrary that $S_{y_{0}}$ is unbounded for some accumulation point $y_{0}$ of $Y$. Set $x_{0}=h^{-1}\left(y_{0}\right)$. Then $x_{0}$ is an accumulation point of $X$. Choose $\varphi_{1} \in \operatorname{Lip}_{\Sigma}(X)$ with values in $[0,1]$ so that $\varphi_{1}\left(x_{0}\right)=1$ and $\varphi_{1}=0$ outside $B\left(x_{0}, 1\right)$. There are $K_{1}<\infty$ and $\sigma_{1} \in \Sigma$ so that $\omega_{\varphi_{1}} \leq K_{1} \sigma_{1}$. Pick a norm-1 vector $u_{1}$ in $E$ and let $f_{1}(x)=\varphi_{1}(x) u_{1}$. Since $T f_{1} \circ h$ is continuous on $X$ and $T f_{1}\left(h\left(x_{0}\right)\right)=S_{y_{0}} u_{1}$ by (1), there exists 
$r_{1} \in(0,1)$ such that $\left\|T f_{1}(h(x))-S_{y_{0}} u_{1}\right\| \leq 1$ for all $x \in B\left(x_{0}, r_{1}\right)$. Choose $x_{1} \in B\left(x_{0}, r_{1}\right) \backslash\left\{x_{0}\right\}$. In general, after $x_{n-1}$ and $\left(u_{m}\right)_{m=1}^{n-1}$ have been determined, let $\varphi_{n} \in \operatorname{Lip}_{\Sigma}(X)$ be a $[0,1]$-valued function such that $\varphi_{n}\left(x_{0}\right)=1$ and $\varphi_{n}=0$ outside $B\left(x_{0}, d\left(x_{n-1}, x_{0}\right)\right)$. There are $K_{n}<\infty$ and $\sigma_{n} \in \Sigma$ so that $\omega_{\varphi_{n}} \leq K_{n} \sigma_{n}$. Of course, $\omega_{\varphi_{n}} \leq 1$ as well. Set $f_{n}(x)=\varphi_{n}(x) u_{n}$ for a vector $u_{n} \in E$ such that $\left\|u_{n}\right\|=\left(n^{2}\left(K_{n}+1\right)\right)^{-1}$ and $\left\|S_{y_{0}} u_{n}\right\| \geq$ $\sum_{m=1}^{n-1}\left\|S_{y_{0}} u_{m}\right\|+2 n$. There exists $r_{n}$ with $0<r_{n}<d\left(x_{n-1}, x_{0}\right) \wedge n^{-1}$ such that $\left\|T f_{n}(h(x))-S_{y_{0}} u_{n}\right\| \leq 1$ for all $x \in B\left(x_{0}, r_{n}\right)$. Choose $x_{n} \in$ $B\left(x_{0}, r_{n}\right) \backslash\left\{x_{0}\right\}$. This completes the inductive construction.

Since $\left\|f_{n}(x)\right\| \leq\left\|u_{n}\right\| \leq 1 / n^{2}$ for all $n$ and all $x \in X, f=\sum f_{n}$ exists. Furthermore, for all $n$,

$$
\omega_{f_{n}} \leq \frac{\omega_{\varphi_{n}}}{n^{2}\left(K_{n}+1\right)} \leq \frac{1}{n^{2}\left(K_{n}+1\right)}\left(K_{n} \sigma_{n} \wedge 1\right) \leq \frac{1}{n^{2}}\left(\sigma_{n} \wedge 1\right) .
$$

By condition (MS2) in the definition of modulus sets, we find $K<\infty$ and $\sigma \in \Sigma$ so that $\sum n^{-2}\left(\sigma_{n} \wedge 1\right) \leq K \sigma$. Hence $\omega_{f} \leq K \sigma$ and thus $f \in \operatorname{Lip}_{\Sigma}(X, E)$. If $m>n$, we have $d\left(x_{n}, x_{0}\right) \geq d\left(x_{m-1}, x_{0}\right)$ and hence $f_{m}\left(x_{n}\right)=0$. For all $n$,

$$
T f\left(h\left(x_{n}\right)\right)=S_{h\left(x_{n}\right)}\left(f\left(x_{n}\right)\right)=\sum_{m=1}^{n} S_{h\left(x_{n}\right)}\left(f_{m}\left(x_{n}\right)\right)=\sum_{m=1}^{n} T f_{m}\left(h\left(x_{n}\right)\right) .
$$

Therefore,

$$
\begin{aligned}
\left\|T f\left(h\left(x_{n}\right)\right)\right\| & \geq\left\|T f_{n}\left(h\left(x_{n}\right)\right)\right\|-\sum_{m=1}^{n-1}\left\|T f_{m}\left(h\left(x_{n}\right)\right)\right\| \\
& \geq\left\|S_{y_{0}} u_{n}\right\|-1-\sum_{m=1}^{n-1}\left(\left\|S_{y_{0}} u_{m}\right\|+1\right) \\
& \geq \sum_{m=1}^{n-1}\left\|S_{y_{0}} u_{m}\right\|+2 n-1-\sum_{m=1}^{n-1}\left(\left\|S_{y_{0}} u_{m}\right\|+1\right)=n .
\end{aligned}
$$

However, $\left(x_{n}\right)$ converges to $x_{0}$ and thus $\left(T f\left(h\left(x_{n}\right)\right)\right)$ converges. We have reached a contradiction.

Now suppose that (2) holds and that $\left(y_{n}\right)$ is an infinite sequence so that $S_{y_{n}}$ is unbounded for all $n$. By the above, each $y_{n}$ is isolated in $Y$,

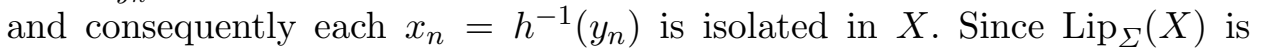
Lipschitz normal, for each $n$, the function $\varphi_{n}$ defined by $\varphi_{n}\left(x_{n}\right)=1$ and $\varphi_{n}(x)=0$ otherwise belongs to $\operatorname{Lip}_{\Sigma}(X)$. Let $K_{n}<\infty$ and $\sigma_{n} \in \Sigma$ be such that $\omega_{\varphi_{n}} \leq K_{n} \sigma_{n}$. For each $n \geq 2$, let $b_{n}=d^{\prime}\left(y_{n}, y_{1}\right)$ and choose $u_{n} \in E$ with $\left\|u_{n}\right\|=\left(n^{2}\left(K_{n}+1\right)\right)^{-1}$ and $\left\|S_{y_{n}} u_{n}\right\|>n \sup _{\sigma \in \Sigma^{\prime}} \sigma\left(b_{n}\right)$. Let $f(x)$ be 
the pointwise sum $\sum_{n=2}^{\infty} \varphi_{n}(x) u_{n}$. Then

$$
\omega_{f} \leq \sum_{n=1}^{\infty} \frac{\omega_{\varphi_{n}}}{n^{2}\left(K_{n}+1\right)} \leq \sum_{n=2}^{\infty} \frac{1}{n^{2}}\left(\sigma_{n} \wedge 1\right) \leq K \sigma_{0}
$$

for some $K<\infty$ and $\sigma_{0} \in \Sigma$. Hence $f \in \operatorname{Lip}_{\Sigma}(X, E)$. It follows that $T f \in \operatorname{Lip}_{\Sigma^{\prime}}(Y, E)$ and so there are $K^{\prime}<\infty$ and $\sigma^{\prime} \in \Sigma^{\prime}$ so that $\omega_{T f} \leq K^{\prime} \sigma^{\prime}$. But $T f\left(y_{n}\right)=S_{y_{n}} u_{n}$ for all $n>1$ and $T f\left(y_{1}\right)=0$. Therefore,

$$
K^{\prime} \sigma^{\prime}\left(b_{n}\right) \geq \omega_{T f}\left(b_{n}\right) \geq\left\|T f\left(y_{n}\right)-T f\left(y_{1}\right)\right\|=\left\|S_{y_{n}} u_{n}\right\|>n \sup _{\sigma \in \Sigma^{\prime}} \sigma\left(b_{n}\right)
$$

for all $n \geq 2$, which is clearly impossible.

REMARK. If $\operatorname{Lip}_{\Sigma^{\prime}}(Y, F)$ consists of bounded functions only, then we may replace $\Sigma^{\prime}$ with $\left\{\sigma \wedge 1: \sigma \in \Sigma^{\prime}\right\}$. In this case, (2) is fulfilled and hence $S_{y}$ is bounded except for finitely many isolated points of $Y$. The special case of the result for spaces of bounded Lipschitz functions was obtained in [6]. Condition (2) also holds for the spaces $\operatorname{Lip}(Y, F)$ and $\operatorname{lip}(Y, F)$.

Let $Y_{1}$ be the set of all $y \in Y$ at which $S_{y}$ is bounded and $X_{1}$ be the set of all $x \in X$ at which $S_{h(x)}^{-1}$ is bounded. The next result is a simple application of the Uniform Boundedness Principle.

Corollary 18. Let $r>0$ be a real number such that $\sigma^{\prime}(r)<\infty$ for all $\sigma^{\prime} \in \Sigma^{\prime}$. Then $\left\{S_{y}: y \in Y_{1}, d\left(y, y_{0}\right) \leq r\right\}$ is uniformly bounded for any $y_{0} \in Y$. Similarly, $\left\{S_{y}: y \in Y_{1}\right\}$ is uniformly bounded if $\sup _{r>0} \sigma^{\prime}(r)<\infty$ for all $\sigma^{\prime} \in \Sigma^{\prime}$.

Proof. For any $u \in E$, there are $K<\infty$ and $\sigma^{\prime} \in \Sigma^{\prime}$ such that $\omega_{T \hat{u}}$ $\leq K \sigma^{\prime}$. If $d\left(y, y_{0}\right) \leq r$, then

$$
\left\|S_{y} u-S_{y_{0}} u\right\|=\left\|T \hat{u}(y)-T \hat{u}\left(y_{0}\right)\right\| \leq K \sigma^{\prime}(r)<\infty .
$$

Hence $\left\{S_{y} u: y \in Y_{1}, d\left(y, y_{0}\right) \leq r\right\}$ is bounded for all $u \in E$. Thus $\left\{S_{y}\right.$ : $\left.y \in Y_{1}, d\left(y, y_{0}\right) \leq r\right\}$ is uniformly bounded by the Uniform Boundedness Principle. The second statement is proved similarly.

In general, we still have "local uniform boundedness".

Proposition 19. For all $y_{0} \in Y_{1}$, there is a neighborhood $V$ of $y_{0}$ in $Y_{1}$ so that $\left\{S_{y}: y \in V\right\}$ is uniformly bounded.

Proof. If the proposition fails, there are sequences $\left(y_{n}\right)$ in $Y_{1}$ converging to $y_{0}$ and $\left(u_{n}\right)$ in $E$ with $\left\|u_{n}\right\|=1 / 2^{n}$ so that $\left\|S_{y_{n}} u_{n}\right\| \rightarrow \infty$. Note that $\left(S_{y_{n}} u_{m}\right)_{m}$ converges to 0 for each $n$ and $\lim _{n} S_{y_{n}} u_{m}=\lim _{n} T \hat{u}_{m}\left(y_{n}\right)=$ $T \hat{u}_{m}\left(y_{0}\right)$ for each $m$. Thus, by passing to subsequences, we may assume that, for each $n,\left\|S_{y_{n}} u_{n}\right\| \geq n+\sum_{m \neq n}\left\|S_{y_{n}} u_{m}\right\|$. Let $w=\sum u_{n}$. Then

$$
\left\|T \hat{w}\left(y_{n}\right)\right\|=\left\|S_{y_{n}} w\right\| \geq\left\|S_{y_{n}} u_{n}\right\|-\sum_{m \neq n}\left\|S_{y_{n}} u_{m}\right\| \geq n
$$


for all $n$. However, $T \hat{w}$ is continuous and so $\left(T \hat{w}\left(y_{n}\right)\right)$ converges, contrary to the above.

Next we consider the continuity of $T$. First we look at a diagonalization lemma.

Lemma 20. Let $\left(g_{n}\right)$ be a sequence of functions from $Y$ into $F$. Suppose that there are a positive sequence $\left(c_{n}\right)$, sequences $\left(y_{1}^{n}\right),\left(y_{2}^{n}\right)$ in $Y$ and $C<\infty$ so that

$$
\begin{aligned}
& \sup _{n} \frac{\left\|g_{n}\left(y_{1}^{n}\right)-g_{n}\left(y_{2}^{n}\right)\right\|}{c_{n}}=\infty, \\
& \sup _{n} \frac{\left\|g_{m}\left(y_{1}^{n}\right)-g_{m}\left(y_{2}^{n}\right)\right\|}{c_{n}}=L_{m}<\infty \quad \text { for all } m, \\
& \sup _{m, n}\left\|g_{m}\left(y_{1}^{n}\right)-g_{m}\left(y_{2}^{n}\right)\right\|=C<\infty .
\end{aligned}
$$

Then there exists a nonnegative summable sequence $\left(\varepsilon_{n}\right)$ so that if the pointwise sum $g=\sum \varepsilon_{n} g_{n}$ converges on the set $\left\{y_{1}^{n}, y_{2}^{n}: n \in \mathbb{N}\right\}$, then $\sup _{n} \| g\left(y_{1}^{n}\right)$ $-g\left(y_{2}^{n}\right) \| / c_{n}=\infty$.

Proof. Let $K_{n}=\left\|g_{n}\left(y_{1}^{n}\right)-g_{n}\left(y_{2}^{n}\right)\right\| / c_{n}$. Choose $n_{1}<n_{2}<\cdots$ and a summable sequence $\left(\varepsilon_{n_{k}}\right)$ so that $\varepsilon_{n_{k}} K_{n_{k}} \geq \max \left\{3 \sum_{m=1}^{k-1} \varepsilon_{n_{m}} L_{n_{m}}, k\right\}$ and $3 C \sum_{m=k+1}^{\infty} \varepsilon_{n_{m}} \leq \varepsilon_{n_{k}} K_{n_{k}} c_{n_{k}}$ for all $k$. Define $\varepsilon_{n}=0$ if $n \neq n_{k}$ for any $k$. If $g=\sum \varepsilon_{n} g_{n}$ converges pointwise on $\left\{y_{1}^{n}, y_{2}^{n}: n \in \mathbb{N}\right\}$, then

$$
\begin{aligned}
\| g\left(y_{1}^{n_{k}}\right) & -g\left(y_{2}^{n_{k}}\right) \| \\
& \geq \varepsilon_{n_{k}}\left\|g_{n_{k}}\left(y_{1}^{n_{k}}\right)-g_{n_{k}}\left(y_{2}^{n_{k}}\right)\right\|-\sum_{m=1}^{k-1} \varepsilon_{n_{m}} L_{n_{m}} c_{n_{k}}-C \sum_{m=k+1}^{\infty} \varepsilon_{n_{m}} .
\end{aligned}
$$

Thus

$$
\left\|g\left(y_{1}^{n_{k}}\right)-g\left(y_{2}^{n_{k}}\right)\right\| \geq \varepsilon_{n_{k}} K_{n_{k}} c_{n_{k}}-\frac{\varepsilon_{n_{k}} K_{n_{k}} c_{n_{k}}}{3}-\frac{\varepsilon_{n_{k}} K_{n_{k}} c_{n_{k}}}{3} \geq \frac{k c_{n_{k}}}{3}
$$

for all $k$.

The next proposition establishes a form of continuity of the operator $T$. For a function $f \in \operatorname{Lip}_{\Sigma}(X, E)$ and a subset $U$ of $X$, let $\|f\|_{U}=\sup \{\|f(x)\|$ : $x \in U\}$.

Proposition 21. Suppose that $U$ is a subset of $X$ such that $V=h(U) \subseteq$ $Y_{1}$ and that $M=\sup \left\{\left\|S_{y}\right\|: y \in V\right\}<\infty$. Then there exists $K<\infty$ so that for any $f \in \operatorname{Lip}_{\Sigma}(X, E)$ with $\omega_{f} \leq \sigma$ for some $\sigma \in \Sigma$ and $\|f\|_{U} \leq 1$, we have $\omega_{T f}^{V} \leq K \sup _{\sigma^{\prime} \in \Sigma^{\prime}} \sigma^{\prime}$, where

$$
\omega_{T f}^{V}(t)=\sup \left\{\left\|T f\left(y_{1}\right)-T f\left(y_{2}\right)\right\|: y_{1}, y_{2} \in V, d^{\prime}\left(y_{1}, y_{2}\right) \leq t\right\} .
$$

Proof. Otherwise, for all $n$, we find $\bar{f}_{n} \in \operatorname{Lip}_{\Sigma}(X, E), \sigma_{n} \in \Sigma$ and $t_{n}>0$ such that $\omega_{\bar{f}_{n}} \leq \sigma_{n},\left\|\bar{f}_{n}\right\|_{U} \leq 1$ and $\sup _{n}\left[\omega_{T \bar{f}_{n}}^{V}\left(t_{n}\right) / \sup _{\sigma^{\prime} \in \Sigma^{\prime}} \sigma^{\prime}\left(t_{n}\right)\right]=\infty$. 
Let $c_{n}=\sup _{\sigma^{\prime} \in \Sigma^{\prime}} \sigma^{\prime}\left(t_{n}\right)$. There are $\left(y_{1}^{n}, y_{2}^{n}\right) \in V \times V$ such that $d^{\prime}\left(y_{1}^{n}, y_{2}^{n}\right) \leq t_{n}$ and that $\sup _{n}\left\|T \bar{f}_{n}\left(y_{1}^{n}\right)-T \bar{f}_{n}\left(y_{2}^{n}\right)\right\| / c_{n}=\infty$. Let $x_{i}^{n}=h^{-1}\left(y_{i}^{n}\right) \in U, i=$ 1,2. Consider the retraction $R: E \rightarrow B_{E}$ as in Lemma 5. Set $f_{n}=R \circ \bar{f}_{n}$. Then $\omega_{f_{n}} \leq 2 \omega_{\bar{f}_{n}} \leq 2 \sigma_{n}$ and $\omega_{f_{n}} \leq 2$. Let $g_{n}=T f_{n}$ for all $n$. Then, for all $m, n$,

$$
\left\|g_{m}\left(y_{1}^{n}\right)-g_{m}\left(y_{2}^{n}\right)\right\|=\left\|S_{y_{1}^{n}} f_{m}\left(x_{1}^{n}\right)-S_{y_{2}^{n}} f_{m}\left(x_{2}^{n}\right)\right\| .
$$

Since $\bar{f}_{n}\left(x_{1}^{n}\right), \bar{f}_{n}\left(x_{2}^{n}\right) \in B_{E}$, we have $S_{y_{i}^{n}} f_{n}\left(x_{i}^{n}\right)=S_{y_{i}^{n}} \bar{f}_{n}\left(x_{i}^{n}\right)=T \bar{f}_{n}\left(x_{i}^{n}\right)$ for $n \in \mathbb{N}, i=1,2$. Thus

$$
\sup _{n} \frac{\left\|g_{n}\left(y_{1}^{n}\right)-g_{n}\left(y_{2}^{n}\right)\right\|}{c_{n}}=\infty .
$$

Since $g_{m} \in \operatorname{Lip}_{\Sigma^{\prime}}(Y, F)$,

$$
\sup _{n} \frac{\left\|g_{m}\left(y_{1}^{n}\right)-g_{m}\left(y_{2}^{n}\right)\right\|}{c_{n}}<\infty \quad \text { for all } m .
$$

Moreover, for all $m$ and $n$,

$$
\left\|g_{m}\left(y_{1}^{n}\right)-g_{m}\left(y_{2}^{n}\right)\right\| \leq M\left(\left\|f_{m}\left(x_{1}^{n}\right)\right\|+\left\|f_{m}\left(x_{2}^{n}\right)\right\|\right) \leq 2 M .
$$

Therefore, Lemma 20 applies to $\left(g_{n}\right)$ and we obtain a summable sequence $\left(\varepsilon_{n}\right)$ as in the lemma. Since $\left\|f_{n}(x)\right\| \leq 1$ for all $n$ and $x, f=\sum \varepsilon_{n} f_{n}$ converges pointwise on $X$. Also,

$$
\omega_{f} \leq \sum \varepsilon_{n} \omega_{f_{n}} \leq \sum \varepsilon_{n}\left(2 \sigma_{n} \wedge 2\right)=2 \sum \varepsilon_{n}\left(\sigma_{n} \wedge 1\right) .
$$

$\operatorname{By}(\operatorname{MS} 2), f \in \operatorname{Lip}_{\Sigma}(X, E)$ and hence $T f \in \operatorname{Lip}_{\Sigma}(X, E)$. Since $S_{y}$ is bounded for all $y \in V$, for all such $y$,

$$
T f(y)=S_{y} f\left(h^{-1}(y)\right)=\sum \varepsilon_{n} S_{y} f_{n}\left(h^{-1}(y)\right)=\sum \varepsilon_{n} g_{n}(y) .
$$

But by the conclusion of Lemma 20, $\sup _{n}\left\|T f\left(y_{1}^{n}\right)-T f\left(y_{2}^{n}\right)\right\| / c_{n}=\infty$, contradicting the fact that $T f \in \operatorname{Lip}_{\Sigma^{\prime}}(Y, F)$.

If $0<\alpha \leq 1$, then $\operatorname{Lip}_{\alpha}(X, E)=\operatorname{Lip}_{\left\{\sigma_{\alpha}\right\}}(X, E)$, where $\sigma_{\alpha}(t)=t^{\alpha}$. Similarly, $\operatorname{lip}_{\alpha}(X, E)=\operatorname{Lip}_{\Sigma}(X, E)$, where $\Sigma$ consists of all modulus functions $\sigma$ such that $\sigma(t) \leq t^{\alpha}$ and $\lim _{t \rightarrow 0} \sigma(t) / t^{\alpha}=0$. Define the $\operatorname{Lip}_{\alpha}$ constant of $f \in \operatorname{Lip}_{\alpha}(X, E)$ to be $L_{\alpha}(f)=\sup \left\{\omega_{f}(t) / t^{\alpha}: t>0\right\}$. For spaces of Lipschitz (Lip) functions, the next corollary was obtained in [6].

Corollary 22. Let $T$ be a biseparating map from $\operatorname{Lip}(X, E)$ onto $\operatorname{Lip}(Y, F)$, respectively from $\operatorname{lip}_{\alpha}(X, E)$ onto $\operatorname{lip}_{\alpha}(Y, F)$. Suppose that $U$ is a bounded subset of $X$ such that $V=h(U)$ is a bounded subset of $Y_{1}$. Then there exists $K<\infty$ so that $L_{1}\left(T f_{\mid V}\right) \leq K\left(L_{1}(f) \vee\|f\|_{U}\right)$ for all $f \in$ $\operatorname{Lip}(X, E)$, respectively, $L_{\alpha}\left(T f_{\mid V}\right) \leq K\left(L_{\alpha}(f) \vee\|f\|_{U}\right)$ for all $f \in \operatorname{lip}_{\alpha}(X, E)$.

Proof. Observe that Proposition 21 applies since $\left\{S_{y}: y \in V\right\}$ is uniformly bounded by Corollary 18 . 
Recall that if $\Sigma$ is a modulus set and $\Sigma_{b}=\{\sigma \wedge 1: \sigma \in \Sigma\}$, then $\operatorname{Lip}_{\Sigma_{b}}(X, E)$ is precisely the space of all bounded functions in $\operatorname{Lip}_{\Sigma}(X, E)$.

Corollary 23. Let $T: \operatorname{Lip}_{\Sigma_{b}}(X, E) \rightarrow \operatorname{Lip}_{\Sigma_{b}^{\prime}}(Y, F)$ be a biseparating map. There exists $K<\infty$ so that for any $f \in \operatorname{Lip}_{\Sigma_{b}}(X, E)$ with $\omega_{f} \leq \sigma \wedge 1$ for some $\sigma \in \Sigma$ and $\|f\|_{X} \leq 1$, we have $\omega_{T f}^{Y_{1}} \leq K \sup _{\sigma^{\prime} \in \Sigma}\left(\sigma^{\prime} \wedge 1\right)$.

Proof. By Corollary 18, $\sup \left\{\left\|S_{y}\right\|: y \in Y_{1}\right\}<\infty$ in this case. The corollary follows immediately from Proposition 21.

Denote by $\mathcal{U}_{b}(X, E)$ the space of bounded uniformly continuous functions from $X$ into $E$.

Corollary 24. Let $T: \mathcal{U}_{b}(X, E) \rightarrow \mathcal{U}_{b}(Y, F)$ be a biseparating map. Then there exists a finite set $I$ of isolated points of $Y$ and $K<\infty$ such that $\sup _{y \in Y \backslash I}\|T f(y)\| \leq K \sup _{x \in X}\|f(x)\|$ for all $f \in \mathcal{U}_{b}(X, E)$.

Proof. Let $\Sigma=\Sigma^{\prime}$ be the set of all modulus functions. Then $\mathcal{U}_{b}(X, E)=$ $\operatorname{Lip}_{\Sigma_{b}}(X, E)$ and $\mathcal{U}_{b}(Y, F)=\operatorname{Lip}_{\Sigma_{b}^{\prime}}(Y, F)$. Recall that $Y_{1}$ is the set of all $y \in Y$ at which $S_{y}$ is bounded. In this case, $I=Y \backslash Y_{1}$ consists of finitely many isolated points of $Y$ by Proposition 17. Fix $x_{0} \in X$ so that $y_{0}=$ $h\left(x_{0}\right) \in Y_{1}$. Suppose that $f \in \mathcal{U}_{b}(X, E)$ with $\sup _{x \in X}\|f(x)\| \leq 1$. By Corollary 23, there exists $K<\infty$ so that for all $y \in Y_{1}$,

$$
\begin{aligned}
\|T f(y)\| & \leq\left\|T f(y)-T f\left(y_{0}\right)\right\|+\left\|T f\left(y_{0}\right)\right\| \\
& \leq \omega_{T f}^{Y_{1}}\left(d^{\prime}\left(y, y_{0}\right)\right)+\left\|S_{y_{0}} f\left(x_{0}\right)\right\| \leq K+\left\|S_{y_{0}}\right\| .
\end{aligned}
$$

We also obtain local continuity of $T$ with respect to the topology of uniform convergence.

Proposition 25. Let $T: \operatorname{Lip}_{\Sigma}(X, E) \rightarrow \operatorname{Lip}_{\Sigma^{\prime}}(Y, F)$ be a biseparating map. If $x_{0} \in X$ and $y_{0}=h\left(x_{0}\right) \in Y_{1}$, then there exists a neighborhood $V$ of $y_{0}$ in $Y_{1}$ and $K<\infty$ so that, setting $U=h^{-1}(V)$, we have $\sup _{y \in V}\|T f(y)\| \leq K$ for all $f \in \operatorname{Lip}_{\Sigma}(X, E)$ such that $\sup _{x \in U}\|f(x)\| \leq 1$.

Proof. By Proposition 19, there is a neighborhood $V$ of $y_{0}$ in $Y_{1}$ such that $\sup \left\{\left\|S_{y}\right\|: y \in V\right\}=K<\infty$. Set $U=h^{-1}(V)$. If $f \in \operatorname{Lip}_{\Sigma}(X, E)$ and $\sup _{x \in U}\|f(x)\| \leq 1$, then for all $y \in V,\|T f(y)\|=\left\|S_{y} f\left(h^{-1}(y)\right)\right\| \leq K$.

We can now deduce the metric properties of the map $h$. For any $x_{1}, x_{2}$ $\in X$, define

$$
s\left(x_{1}, x_{2}\right)=\sup \left\{\left\|f\left(x_{2}\right)\right\|: f\left(x_{1}\right)=0, \omega_{f} \leq \sigma \text { for some } \sigma \in \Sigma\right\} .
$$

Proposition 26. Let $U$ be a subset of $X_{1}$ so that $V=h(U)$ is a subset of $Y_{1}$. Assume $\sup \left\{\left\|S_{y}\right\|,\left\|S_{y}^{-1}\right\|: y \in V\right\}=M<\infty$ and that $\sup \left\{s\left(x_{1}, x_{2}\right): x_{1}, x_{2} \in U\right\}=C<\infty$. There exists $K<\infty$ such that $s\left(x_{1}, x_{2}\right) \leq K \sup _{\sigma^{\prime} \in \Sigma^{\prime}} \sigma^{\prime}\left(d^{\prime}\left(h\left(x_{1}\right), h\left(x_{2}\right)\right)\right)$ for all $x_{1}, x_{2} \in U$. 
Proof. Appeal to Proposition 21 to find $K<\infty$ so that for any $f \in$ $\operatorname{Lip}_{\Sigma}(X, E)$ with $\omega_{f} \leq \sigma$ for some $\sigma \in \Sigma$ and $\|f\|_{U} \leq 1$, we have $\omega_{T f}^{V} \leq$ $K \sup _{\sigma^{\prime} \in \Sigma^{\prime}} \sigma^{\prime}$. If $a<s\left(x_{1}, x_{2}\right), x_{1}, x_{2} \in U$, choose $f \in \operatorname{Lip}_{\Sigma}(X, E)$ and $\sigma \in \Sigma$ so that $f\left(x_{1}\right)=0,\left\|f\left(x_{2}\right)\right\|>a$ and $\omega_{f} \leq \sigma$. Now $x \in U$ implies that $\|f(x)\| \leq s\left(x_{1}, x\right) \leq C$. Let $\bar{f}=f /(C \vee 1)$. Then $\omega_{\bar{f}} \leq \sigma$ and $\|\bar{f}\|_{U} \leq 1$. Thus

$$
\begin{aligned}
\frac{a}{C \vee 1} & <\left\|\bar{f}\left(x_{2}\right)\right\| \leq M\left\|S_{h\left(x_{2}\right)} \bar{f}\left(x_{2}\right)\right\|=M\left\|T \bar{f}\left(h\left(x_{1}\right)\right)-T \bar{f}\left(h\left(x_{2}\right)\right)\right\| \\
& \leq M \omega_{T \bar{f}}^{V}\left(d^{\prime}\left(h\left(x_{1}\right), h\left(x_{2}\right)\right)\right) \leq M K \sup _{\sigma^{\prime} \in \Sigma^{\prime}} \sigma^{\prime}\left(d^{\prime}\left(h\left(x_{1}\right), h\left(x_{2}\right)\right)\right) .
\end{aligned}
$$

This proves the proposition.

Corollary $27([6])$. Let $T: \operatorname{Lip}(X, E) \rightarrow \operatorname{Lip}(Y, F)$ be a biseparating map and $h: X \rightarrow Y$ be the homeomorphism associated to T. For any bounded set $U \subseteq X$ such that $V=h(U)$ is bounded in $Y, h$ is Lipschitz on $U$ and $h^{-1}$ is Lipschitz on $V$.

Proof. By Proposition 17, the set $Y_{1}$ consisting of all $y \in Y$ where $S_{y}$ is bounded contains all of $\bar{Y}$ except for finitely many isolated points of $Y$. A similar statement holds for $X_{1}$. Thus it suffices to show that $h$ is Lipschitz on $U_{1}=U \cap X_{1} \cap h^{-1}\left(Y_{1}\right)$ and that $h^{-1}$ is Lipschitz on $V_{1}=h\left(U_{1}\right)$. By Corollary $18, \sup \left\{\left\|S_{y}\right\|,\left\|S_{y}^{-1}\right\|: y \in V_{1}\right\}<\infty$. For all $x_{1}, x_{2} \in X$, it is clear that the quantity $s\left(x_{1}, x_{2}\right)$ defined above has the value $d\left(x_{1}, x_{2}\right)$. In particular, $\sup \left\{s\left(x_{1}, x_{2}\right): x_{1}, x_{2} \in V_{1}\right\}<\infty$ since $V_{1}$ is bounded. By Proposition 26, there exists $K<\infty$ such that $d\left(x_{1}, x_{2}\right) \leq K d^{\prime}\left(h\left(x_{1}\right), h\left(x_{2}\right)\right)$ for all $x_{1}, x_{2} \in U_{1}$. This shows that $h^{-1}$ is Lipschitz on $V_{1}$. The proof for $h$ is similar.

REMARK. If $0<\alpha<1$, then for a bounded subset $U$ of the space $\operatorname{lip}_{\alpha}(X, E)$, there is a positive constant $K_{\alpha}$ so that $s\left(x_{1}, x_{2}\right) \geq K_{\alpha} d^{\alpha}\left(x_{1}, x_{2}\right)$ for all $x_{1}, x_{2} \in U$. Hence Corollary 27 also applies to biseparating maps between $\operatorname{lip}_{\alpha}$ spaces, $0<\alpha<1$.

Proposition 28. Let $T: \operatorname{Lip}_{\Sigma}(X, E) \rightarrow \operatorname{Lip}_{\Sigma^{\prime}}(Y, F)$ be a biseparating map. Suppose that $\operatorname{Lip}(Y, F) \subseteq \operatorname{Lip}_{\Sigma^{\prime}}(Y, F)$. If $U$ is a subset of $X$ so that $V=h(U) \subseteq Y_{1}$ and $\sup _{y \in V}\left\|S_{y}\right\|<\infty$, then $h$ is uniformly continuous on $U$.

Proof. If the proposition fails, we can find a sequence $\left(\left(x_{1}^{n}, x_{2}^{n}\right)\right)_{n}$ in $U \times U$ and an $\varepsilon>0$ so that $\lim d\left(x_{1}^{n}, x_{2}^{n}\right)=0$ and $d^{\prime}\left(y_{1}^{n}, y_{2}^{n}\right) \geq \varepsilon$ for all $n$, where $y_{i}^{n}=h\left(x_{i}^{n}\right)$. If $\left(y_{1}^{n}\right)$ has a convergent subsequence, then by continuity of $h^{-1}$ and the fact that $\lim d\left(x_{1}^{n}, x_{2}^{n}\right)=0$, there is a subsequence $I$ of $\mathbb{N}$ so that $\left(x_{1}^{n}\right)_{n \in I}$ and $\left(x_{2}^{n}\right)_{n \in I}$ both converge to the same $x_{0}$. Then $\left(y_{1}^{n}\right)_{n \in I}$ and $\left(y_{2}^{n}\right)_{n \in I}$ must both converge to $h\left(x_{0}\right)$, contrary to their choice. Thus $\left(y_{1}^{n}\right)$, and, by symmetry, $\left(y_{2}^{n}\right)$ cannot have convergent subsequences. Without loss 
of generality, there exists $\delta$ with $0<4 \delta<\varepsilon$ such that $d\left(y_{1}^{n}, y_{1}^{m}\right), d\left(y_{2}^{n}, y_{2}^{m}\right)$ $>2 \delta$ for all $m \neq n$. Then the sets $B\left(y_{1}^{n}, \delta\right), n \in \mathbb{N}$, are pairwise disjoint and each can contain at most one $y_{2}^{m}$, in which case $m \neq n$. Hence we can choose a subsequence $J$ of $\mathbb{N}$ so that $y_{2}^{n} \notin B\left(y_{1}^{m}, \delta\right)$ if $n, m \in J$. Pick a normalized vector $v \in F$ and let $g(y)=v \cdot \sup _{n \in J}\left(\delta-d\left(y, y_{1}^{n}\right)\right)^{+}$. Then $g \in \operatorname{Lip}(Y, F) \subseteq$ $\operatorname{Lip}_{\Sigma^{\prime}}(Y, F)$ and hence $f=T^{-1} g \in \operatorname{Lip}_{\Sigma}(X, E)$. In particular, $f$ is uniformly continuous. However, for all $n \in J$,

$$
\left\|f\left(x_{1}^{n}\right)-f\left(x_{2}^{n}\right)\right\|=\left\|S_{y_{1}^{n}}^{-1} g\left(y_{1}^{n}\right)-S_{y_{2}^{n}}^{-1} g\left(y_{2}^{n}\right)\right\|=\delta\left\|S_{y_{2}^{n}}^{-1} v\right\| \geq \delta\left\|S_{y_{2}^{n}}\right\|^{-1} .
$$

Since $\sup \left\|S_{y_{2}^{n}}\right\|<\infty$ and $d\left(x_{1}^{n}, x_{2}^{n}\right) \rightarrow 0, f$ cannot be uniformly continuous.

Acknowledgements. The author thanks Wee-Kee Tang for many stimulating conversations regarding the material contained herein.

This research was partially supported by AcRF project no. R-146-000130-112.

\section{References}

[1] J. Araujo, Realcompactness and spaces of vector-valued continuous functions, Fund. Math. 172 (2002), 27-40.

[2] -, Realcompactness and Banach-Stone theorems, Bull. Belg. Math. Soc. Simon Stevin 11 (2004), 247-258.

[3] - Linear biseparating maps between spaces of vector-valued differentiable functions and automatic continuity, Adv. Math. 187 (2004), 488-520.

[4] - The noncompact Banach-Stone theorem, J. Operator Theory 55 (2006), 285-294.

[5] J. Araujo, E. Beckenstein and L. Narici, Biseparating maps and homeomorphic realcompactifications, J. Math. Anal. Appl. 192 (1995), 258-265.

[6] J. Araujo and L. Dubarbie, Biseparating maps between Lipschitz function spaces, ibid. 357 (2009), 191-200.

[7] J. Araujo and K. Jarosz, Separating maps on spaces of continuous functions in: Function Spaces (Edwardsville, IL, 1998), Contemp. Math. 232, Amer. Math. Soc., Providence, RI, 1999, 33-37.

[8] —, - Automatic continuity of biseparating maps, Studia Math. 155 (2003), 231239.

[9] S. Banach, Théorie des Opérations Linéaires, Warszawa, 1932; reprinted by Chelsea, New York, 1963.

[10] E. Behrends, M-structure and the Banach-Stone Theorem, Springer, Berlin, 1978.

[11] R. J. Fleming and J. E. Jamison, Isometries on Banach Spaces, Vols. 1 and 2, Chapman \& Hall/CRC Monogr. Surv. Pure Appl. Math. 129 and 138, Chapman \& Hall/CRC, Boca Raton, FL, 2003 and 2008.

[12] M. I. Garrido and J. A. Jaramillo, A Banach-Stone theorem for uniformly continuous functions, Monatsh. Math. 131 (2000), 189-192.

[13] - , - Variations on the Banach-Stone theorem, Extracta Math. 17 (2002), 351383.

[14] —, -, Lipschitz-type functions on metric spaces, J. Math. Anal. Appl. 340 (2008), $282-290$. 
[15] H. L. Gau, J.-S. Jeang and N.-C. Wong, Biseparating linear maps between continuous vector-valued function spaces, J. Austral. Math. Soc. 74 (2003), 101-109.

[16] I. Gelfand and A. Kolmogorov, On rings of continuous functions on topological spaces, Dokl. Akad. Nauk SSSR 22 (1939), 11-15.

[17] L. Gillman and M. Jerison, Rings of Continuous Functions, Grad. Texts in Math. 43, Springer, New York, 1976.

[18] J.-S. Jeang and Y.-F. Lin, Characterizations of disjointness preserving operators on vector-valued function spaces, Proc. Amer. Math. Soc. 136 (2008), 947-954.

[19] A. Jiménez-Vargas, Linear bijections preserving the Hölder seminorm, ibid. 135 (2007), 2539-2547.

[20] —, Disjointness preserving operators between little Lipschitz algebras, J. Math. Anal. Appl. 337 (2008), 984-993.

[21] A. Jiménez-Vargas and M. Villegas-Vallecillos, Order isomorphisms of little Lipschitz algebras, Houston J. Math. 34 (2008), 1185-1195.

[22] A. Jiménez-Vargas, M. Villegas-Vallecillos and Y.-S. Wang, Banach-Stone theorems for vector-valued little Lipschitz functions, Publ. Math. Debrecen 74 (2009), 81-100.

[23] A. Jiménez-Vargas and Y.-S. Wang, Linear biseparating maps between vector-valued little Lipschitz function spaces, preprint.

[24] J. A. Johnson, Banach spaces of Lipschitz functions and vector-valued Lipschitz functions, Trans. Amer. Math. Soc. 148 (1970), 147-169.

[25] I. Kaplansky, Lattices of continuous functions, Bull. Amer. Math. Soc. 53 (1947), 617-623.

[26] K. de Leeuw, Banach spaces of Lipschitz functions, Studia Math. 21 (1961/62), $55-66$.

[27] Y.-F. Lin and N.-C. Wong, The structure of compact disjointness preserving operators on continuous functions, Math. Nachr. 282 (2009), 1009-1021.

[28] S. MacLane and G. Birkhoff, Algebra, 2nd ed., Macmillan, New York, 1979.

[29] D. Sherbert, Banach algebras of Lipschitz functions, Pacific J. Math. 13 (1963), 1387-1399.

[30] - , The structure of ideals and point derivations in Banach algebras of Lipschitz functions, Trans. Amer. Math. Soc. 111 (1964), 240-272.

[31] M. H. Stone, Applications of the theory of Boolean rings to general topology, ibid. 41 (1937), 375-481.

[32] N. Weaver, Lipschitz Algebras, World Sci., Singapore, 1999.

Denny H. Leung

Department of Mathematics

National University of Singapore

2 Science Drive 2

Singapore 117543

E-mail: matlhh@nus.edu.sg

Received November 18, 2008

Revised version June 1, 2009 